01

\title{
Моделирование коэффициента теплопроводности наножидкости с малыми частицами методом молекулярной динамики
}

\author{
(С) В.Я. Рудяк, С.Л. Краснолуцкий \\ Новосибирский государственный архитектурно-строительный университет (Сибстрин), \\ 630008 Новосибирск, Россия \\ e-mail: valery.rudyak@mail.ru
}

(Поступило в Редакцию 11 июля 2016 г.)

\begin{abstract}
Методом молекулярной динамики выполнено моделирование коэффициента теплопроводности наножидкостей. Рассмотрены наножидкости на основе аргона с частицами из алюминия и цинка размером от 1 до $4 \mathrm{~nm}$. Объемная концентрация наночастиц менялось от 1 до 5\%. Изучена зависимость коэффициента теплопроводности от объемной концентрации наночастиц. Показано, что теплопроводность наножидкости не описывается классическими теориями. В частности, она зависит от размера частиц и растет с его увеличением. Однако установлено, что у наножидкостей с малыми частицами теплопроводность может быть даже ниже, чем теплопроводность базовой жидкости. Систематически изучено поведение корреляционных функций, ответственных за теплопроводность и качественно объясняется причина роста теплопроводности наножидкостей.
\end{abstract}

DOI: $10.21883 /$ JTF.2017.10.44986.1981

\section{Введение}

Хорошо известно, что теплопроводность твердых тел, в частности, металлов, их окислов, графита и его производных на несколько порядков превосходит теплопроводность обычно использующихся жидких теплоносителей (воды, этиленгликоля, различных фреонов и т.п.). Идея использовать дисперсные жидкости в качестве теплоносителей и хладагентов родилась давно, однако традиционные дисперсные жидкости применить с этой целью не удалось из-за седиментации дисперсных частиц и их абразивности. Дисперсные же жидкости с наночастицами, названные наножидкостями, не имеют этих недостатков. Уже первые эксперименты по измерению их теплопроводности $[1,2]$ показали прекрасные результаты: добавление даже малых (порядка долей процента) концентраций металлических наночастиц существенно повышало теплопроводность базовой жидкости. По этой причине наножидкости с успехом могут применяться для охлаждения машин и агрегатов, в частности, в электронных системах, в теплоэнергетике при транспортировке тепловой энергии и т. п. В 2008 году Роутбортом с коллегами был разработан проект по применению наножидкостей в качестве хладогентов в промышленных установках (см. также [3]).

Поскольку во всех приложениях имеют место течения наножидкостей, то, чтобы управлять соответствующими процессами, необходимы достоверные знания относительно теплофизических характеристик наножидкостей. Сегодня уже ясно, что они не стандартны и не описываются классическими теориями для крупнодисперсных жидкостей. С вязкостью такое понимание практически достигнуто (см., например, работы [4-9] и цитированную там литературу). Показано (и экспериментально, и молекулярно-динамическим моделированием), что вяз- кость наножидкостей существенно превосходит вязкость крупнодисперсных жидкостей при той же объемной концентрации наночастиц. Однако в отличие от последних она зависит от размера частиц и их материала, причем вязкость тем больше, чем меньше размер частиц. В общем понятны и причины указанных особенностей, хотя универсальных соотношений все еще нет, да их и трудно ожидать.

С теплопроводностью наножидкостей ситуация более сложная, несмотря на то что ее измерениям посвящено огромное число работ. Сегодня установлено, что теплопроводность наножидкостей с ростом концентрации частиц выходит на некоторый предельный уровень $[10,11]$. Кроме того, она также зависит от размера наночастиц и увеличивается с его ростом. Относительно величины превышения коэффициента теплопроводности над соответствующим значением для базовой жидкости экспериментальные данные весьма противоречивы. Обычно указывается, что оно зависит от концентрации и практически всегда выше, чем это предсказывает теория Максвелла

$$
\lambda=\lambda_{f}\left[1+\frac{3(1-\varpi) \varphi}{1+2 \varpi-\varphi(1-\varpi)}\right],
$$

где $\varpi=\lambda_{f} / \lambda_{p}, \lambda_{f}$ и $\lambda_{p}-$ коэффициент теплопроводности базовой жидкости и материала частиц, $\varphi-$ их объемная концентрация. Однако если в наножидкостях коэффициент теплопроводности растет с увеличением размера частиц, то возникает вопрос: а каков он в наножидкостях с самыми малыми частицами?

Исследованию этого вопроса и посвящена настоящая работа. Экспериментально ответить на него чрезвычайно трудно, поскольку с уменьшением размера частиц их числовая плотность быстро растет (при заданной объемной концентрации она обратно пропорциональна 
кубу радиуса частицы). Это приводит к их активному взаимодействию, сопровождающемуся образованием конгломератов. В результате существенно меняется средний размер частиц и адекватность интерпретации полученных данных. Чтобы избежать агломерации, обычно используют те или иные поверхностно активные вещества (ПАВ). Но ПАВ существенно меняет теплофизические свойства наножидкости и тем сильнее, чем меньше размер наночастиц. С другой стороны, метод молекулярной динамики, имея предсказательную силу эксперимента, позволяет реализовать наиболее чистый эксперимент, в частности, использовать монодисперсные частицы. В настоящей работе поэтому именно этот метод и используется.

Существует и еще одна важная причина применения метода молекулярной динамики. Коэффициент теплопроводности наножидкости является некоторым интегральным свойством этой нестандартной двухфазной системы. Экспериментальное его изучение практически не позволяет выявить механизмы, определяющие теплопроводность наножидкостей, удается получить лишь достаточно грубую информацию о его зависимости от тех или иных параметров (объемной концентрации частиц, их размера и т.п.). К настоящему времени построено много феноменологических моделей теплопроводности наножидкостей, в основу которых положены различные модельные представления, например, о влиянии броуновского движения частиц и т.п. (см. обзор [12]). Все они чрезвычайно остроумны, но базируются обычно лишь на косвенных физических соображениях относительно механизмов переноса в наножидкостях. Метод же молекулярной динамики позволяет в явном виде изучить и проанализировать эти механизмы и создать тем самым прочную базу для построения адекватных макроскопических моделей теплопроводности наножидкостей.

Ранее уже проводилось молекулярно-динамическое моделирование с раздельным учетом различных вкладов в коэффициент теплопроводности наножидкости. В частности, в [13] было проведено моделирование теплопроводности наножидкости на основе ксенона с добавлением нанокластеров из атомов платины. Однако работа имеет ряд методических недостатков, ее авторы сами признают, что рассчитывали коэффициент теплопроводности наножидкости, которая является бинарной системой, по формуле для однокомпонентных систем и без учета диффузионного вклада.

В настоящей работе рассматривались модельные наножидкости, несущим флюидом в которых является аргон, со сферическими наночастицами алюминия и цинка. Размер наночастиц варьировал от 1 до $4 \mathrm{~nm}$, их объемная концентрация - от 1 до 5\%.

\section{Методика моделирования}

Для моделирования применялся стандартный метод молекулярной динамики. Использовался оригинальный пакет SibMD, ранее применявшийся при решении различных задач теории переноса наножидкостей [14-16]. Моделирование проводилось в кубической ячейке с периодическими граничными условиями. Взаимодействие атомов несущей среды между собой определялось потенциалом Леннард-Джонса

$$
\Phi_{L J}(r)=4 \varepsilon\left\lfloor(\sigma / r)^{12}-(\sigma / r)^{6}\right\rfloor,
$$

где $\sigma$ - эффективный диаметр молекулы среды, $\varepsilon-$ глубина потенциальной ямы, $r=\left|\mathbf{r}_{i}-\mathbf{r}_{j}\right|-$ расстояние между центрами молекул $i$ и $j$.

Взаимодействие молекул несущей среды с наночастицей — потенциалом РК [17]

$$
\begin{gathered}
\Psi(r)=\Psi_{9}(r)-\Psi_{3}(r), \\
\Psi_{i}=C_{i}\left\{\left[\frac{1}{(r-R)^{i}}-\frac{1}{(r+R)^{i}}\right]\right. \\
\left.-\frac{a_{i}}{r}\left[\frac{1}{(r-R)^{i-1}}-\frac{1}{(1+R)^{i-1}}\right]\right\},
\end{gathered}
$$

где $i=9,3, a_{9}=9 / 8, a_{5}=3 / 2, C_{9}=\left(4 \pi \varepsilon_{12} \sigma_{12}^{12}\right) / 45 V_{p}$, $C_{3}=\left(2 \pi \varepsilon_{12} \sigma_{12}^{6}\right) / 3 V_{p}, V_{p}^{-1}=\rho_{p} / m_{p}$. Здесь $\rho_{p}-$ плотность материала наночастицы, $m_{p}$ - масса атома вещества, из которого состоит наночастица, $R$ - радиус наночастицы, $\sigma_{i j}, \varepsilon_{i j}$ - параметры потенциала (2) взаимодействия молекулы несущей жидкости с атомом наночастицы.

В качестве потенциала взаимодействия наночастиц используется потенциал [18], который для монодисперсных наночастиц имеет вид

$$
\begin{gathered}
U(r, R)=U_{7}(r, R)-U_{1}(r, R), \\
U_{7}(r, R)=\frac{\pi^{2}}{315} \frac{\tilde{\varepsilon} \tilde{\sigma}^{12}}{V_{p}^{2}}\left\{\frac{R^{2}}{r}\left[\frac{1}{(r-2 R)^{7}}+\frac{2}{r^{7}}+\frac{1}{(r+2 R)^{7}}\right]\right. \\
-\frac{R}{3 r}\left[\frac{1}{(r-2 R)^{6}}-\frac{1}{(r+2 R)^{6}}\right] \\
\left.-\frac{1}{30 r}\left[\frac{1}{(r-2 R)^{5}}-\frac{2}{r^{5}}+\frac{1}{(r+2 R)^{5}}\right]\right\}, \\
U_{1}(r, R)=\frac{2 \pi^{2}}{3} \frac{\tilde{\varepsilon} \tilde{\sigma}^{6}}{V_{p}^{2}}\left[\ln \left(\frac{r^{2}-4 R^{2}}{r^{2}}\right)\right. \\
\left.+2 R^{2}\left(\frac{1}{r^{2}-4 R^{2}}+\frac{1}{r^{2}}\right)\right] .
\end{gathered}
$$

Здесь $R$ - радиус наночастиц, $\tilde{\varepsilon}$ и $\tilde{\sigma}-$ параметры потенциала Леннард-Джонса (2) взаимодействия атомов наночастицы. Потенциалы (3) и (4) построены в предположении, что взаимодействие атомов (молекул) несущей среды с атомами наночастиц и атомов наночастиц между собой описываются потенциалом вида (1) соответственно с параметрами $\sigma_{12}, \varepsilon_{12}$ и $\tilde{\sigma}, \tilde{\varepsilon}$. 
Моделирование проводилось следующим образом. Молекулы среды и наночастицы равномерно размещались в ячейке моделирования в соответствии с заданными значениями плотности несущей среды и объемной концентрации наночастиц. Плотность базовой жидкости определялась вириальным параметром $\rho=N \sigma^{3} / V$, объем ячейки моделирования для наножидкости - соотношением: $V=\alpha N_{1} \sigma^{3} / \sqrt{2}+4 \pi \beta N_{2} R^{3} / 3$, где $N_{1}$ и $N_{2}-$ число молекул флюида и наночастиц соответственно, а $\alpha=V / V_{0}, V_{0}=N \sigma^{3} / \sqrt{2}$ - объем плотной упаковки $N$ молекул. При добавлении наночастиц коэффициент $\beta$ подбирался так, чтобы давление наножидкости было равно давлению базовой жидкости с заданной плотностью.

Начальные скорости молекул задавались в соответствии с распределением Максвелла при заданной температуре, наночастиц - равными нулю. Расчет начинался после начального периода релаксации, когда вся система приходила в равновесие. Для интегрирования уравнений Ньютона применялась схема Верле.

Поскольку используемые потенциалы имеют бесконечный радиус действия, в процессе моделирования их необходимо обрезать. Для потенциала (2) радиус обрезания равнялся $2.5 \sigma$, а для потенциала (3) он подбирался таким образом, чтобы на радиусе обрезания для потенциала (3) сила, действующая на атом аргона со стороны наночастицы, была равна силе взаимодействия двух молекул на радиусе обрезания для потенциала (2). Потенциал наночастиц обрезался на расстоянии, при котором сила взаимодействия наночастиц равна нулю, т.е. фактически использовался так называемый потенциал мягких сфер.

Параметры потенциала взаимодействия молекул аргона были следующими: $\sigma=3.405 \AA, \varepsilon / k_{B}=119.8 \mathrm{~K}[19]$. Для расчета параметров потенциалов (3) и (4) использовались следующие параметры потенциала (2): для цинка $\alpha=2.46 \AA, \varepsilon / k_{B}=1040 \mathrm{~K}[20]$, для алюминия $\sigma=2.551 \AA, \varepsilon / k_{B}=857.6 \mathrm{~K}$. Последние параметры были получены на основе данных по модулю Юнга и строению кристаллической решетки (кубическая гранецентрированная) методом, аналогичным описанному в работе [21]. Параметры $\sigma_{12}, \varepsilon_{12}$ определялись с помощью простейших комбинационных соотношений $\sigma_{12}=\sqrt{\sigma \tilde{\sigma}}$, $\varepsilon_{12}=\sqrt{\varepsilon \tilde{\varepsilon}}$.

Поскольку при молекулярно-динамическом расчете фазовых траекторий системы имеет место их локальная неустойчивость и перемешивание [22,23], полученные данные необходимо усреднять по ансамблю независимых фазовых траекторий. В настоящей работе усреднение проводилось по тысяче независимых фазовых траекторий.

Коэффициент теплопроводности наножидкости (бинарной смеси) рассчитывался на основе флуктуационнодиссипационной теоремы, связывающей коэффициент теплопроводности с корреляционной функцией соответствующих динамических переменных (такие формулы принято называть формулами Грина-Кубо). Сложность вычисления коэффициента теплопроводности для рассматриваемой бинарной системы состоит в том, что необходимо исключить диффузионный поток тепла. Если это сделать, то коэффициент теплопроводности наножидкости будет иметь вид [24]

$$
\lambda=\lambda_{0}-\lambda_{d} \equiv \frac{L_{0}}{T^{2}}-\frac{L_{2}^{2}}{L_{22} T^{2}} .
$$

Входящие сюда коэффициенты равны интегралам от равновесных корреляционных функций

$$
\begin{gathered}
L_{0}=\frac{V}{3} \int_{0}^{\tau}\left\langle\mathbf{j}_{Q}(0) \mathbf{j}_{Q}(t)\right\rangle d t, \\
L_{22}=\frac{V}{3} \int_{0}^{\tau}\left\langle\mathbf{j}_{d 2}(0) \mathbf{j}_{d 2}(t)\right\rangle d t, \\
L_{2}=\frac{V}{6} \int_{0}^{\tau}\left\langle\mathbf{j}_{Q}(0) \mathbf{j}_{d 2}(t)\right\rangle d t+\frac{V}{6} \int_{0}^{\tau}\left\langle\mathbf{j}_{d 2}(0) \mathbf{j}_{Q}(t)\right\rangle d t .
\end{gathered}
$$

Угловые скобки в (6),(7) означают усреднение по ансамблю, а $\tau$ - время выхода на платовое значение [25]. Входящие сюда диффузионный поток наночастиц (второго компонента), $\mathbf{j}_{d 2}$, и поток тепла, $\mathbf{j}_{Q}$, определяются соотношениями

$$
\mathbf{j}_{d 2}(t)=\frac{1}{V} \sum_{\alpha=2, i=1}^{N_{2}} \mathbf{v}_{2, i}(t), \quad \mathbf{j}_{Q}(t)=\mathbf{j}_{Q}^{\mathrm{K}}(t)+\mathbf{j}_{Q}^{\mathrm{P}}(t)+\mathbf{j}_{Q}^{\mathrm{C}}(t) .
$$

В последнюю формулу входят три различных члена, $\mathbf{j}_{Q}^{K}, \mathbf{j}_{Q}^{P}, \mathbf{j}_{Q}^{C}$, задающих соответственно поток тепла, обусловленный переносом кинетической и потенциальной энергий, и поток тепла, возникающий в результате столкновений. Эти вклады определяются так

$$
\begin{aligned}
\mathbf{j}_{Q}^{\mathrm{K}}(t)= & \frac{1}{V}\left[\frac{m_{1}}{2} \sum_{\alpha=1, i=1}^{N_{1}} \mathbf{v}_{1, i}(t) v_{1, i}^{2}(t)\right. \\
& \left.+\frac{m_{2}}{2} \sum_{\alpha=2, i=1}^{N_{2}} \mathbf{v}_{2, i}(t) v_{2, i}^{2}(t)\right], \\
\mathbf{j}_{Q}^{\mathrm{P}}(t)= & \frac{1}{V}\left[\frac{1}{2} \sum_{\alpha=1, i=1}^{N_{1}} \sum_{\alpha=1, j \neq i} \mathbf{v}_{1, i}(t) \Phi_{11, i j}\left(r_{i j}(t)\right)\right. \\
& +\frac{1}{2} \sum_{\alpha=1, i=1}^{N_{1}} \sum_{\alpha=2, j=i}^{N_{2}} \mathbf{v}_{1, i}(t) \Phi_{12, i j}\left(r_{i j}(t)\right) \\
& +\frac{1}{2} \sum_{\alpha=2, i=1}^{N_{2}} \sum_{\alpha=1, j=i}^{N_{1}} \mathbf{v}_{2, i}(t) \Phi_{12, i j}\left(r_{i j}(t)\right) \\
& \left.+\frac{1}{2} \sum_{\alpha=2, i=1}^{N_{2}} \sum_{\alpha=2, j \neq i} \mathbf{v}_{2, i}(t) \Phi_{22, i j}\left(r_{i j}(t)\right)\right]
\end{aligned}
$$




$$
\begin{aligned}
\mathbf{j}_{Q}^{\mathrm{C}}(t)= & \frac{1}{V}\left[\frac{1}{2} \sum_{\alpha=1, i=1}^{N_{1}} \sum_{\alpha=1, j \neq i} \mathbf{r}_{i j}(t)\left(\mathbf{F}_{11, i j}(t) \mathbf{v}_{1, i}(t)\right)\right. \\
& +\frac{1}{2} \sum_{\alpha=1, i=1}^{N_{1}} \sum_{\alpha=2, j=i}^{N_{2}} \mathbf{r}_{i j}(t)\left(\mathbf{F}_{12, i j}(t) \mathbf{v}_{2, j}(t)\right) \\
& +\frac{1}{2} \sum_{\alpha=2, i=1}^{N_{2}} \sum_{\alpha=1, j=i}^{N_{1}} \mathbf{r}_{i j}(t)\left(\mathbf{F}_{12, i j}(t) \mathbf{v}_{1, j}(t)\right) \\
& \left.+\frac{1}{2} \sum_{\alpha=2, i=1}^{N_{2}} \sum_{\alpha=2, j \neq i} \mathbf{r}_{i j}(t)\left(\mathbf{F}_{22, i j}(t) \mathbf{v}_{2, j}(t)\right)\right]
\end{aligned}
$$

Здесь индекс $\alpha=1$ относится к атомам аргона, $2-$ к наночастицам, $m_{i}$ - масса частицы (атома или наночастицы), $V$ - объем системы, $T$ - температура среды, $\Phi_{11, i j}$ - потенциалы взаимодействия атомов базовой жидкости, $\Phi_{12, i j}$ - ее атомов и частиц, $\Phi_{22, i j}$ - частиц, а $\mathbf{F}_{\alpha \beta, i j}$ - соответствующие силы. $N_{1}$ и $N_{2}$ - число атомов аргона и наночастиц соответственно.

\section{Результаты моделирования}

Согласно (5)-(7), моделируемый коэффициент теплопроводности является функцией времени. Собственно значение коэффициента теплопроводности получается, когда время интегрирования достигает платового значения $\tau$, начиная с которого он перестает меняться. Эволюция функции $\lambda(t)(5)$, определяющей коэффициент теплопроводности, представлена на рис. 1 для наножидкости с частицами цинка размером $2 \mathrm{~nm}$. Объемная концентрация частиц составляла $4.2 \%$, а массовая 0.208. Здесь время измеряется в единицах $\tau_{0}=\sigma / c$, где $c$ - тепловая скорость молекул несущей среды. Выход на платовое значение достигается на временах около $100 \tau_{0}$.

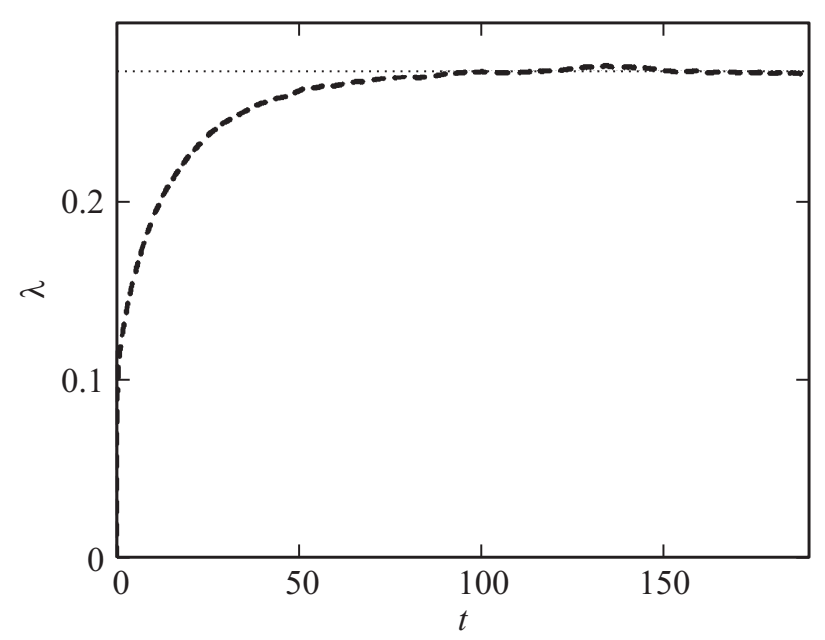

Рис. 1. Эволюция коэффициента теплопроводности наножидкости $\mathrm{Ar}-\mathrm{Zn}, \mathrm{W} /(\mathrm{mK}), d=2 \mathrm{~nm}, \varphi=4.2 \%$.

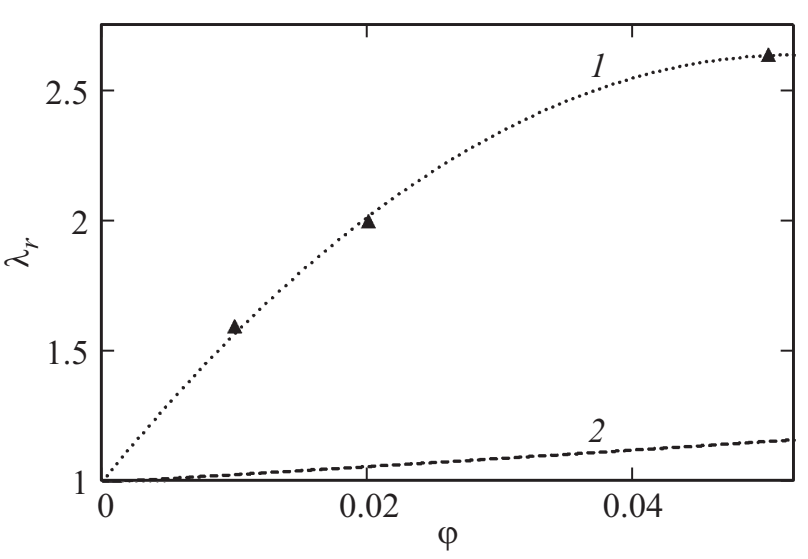

Рис. 2. Зависимость относительного коэффициента теплопроводности наножидкости $\mathrm{Ar}-\mathrm{Zn}$ от объемной концентрации наночастиц $(d=2 \mathrm{~nm})$.

Типичная зависимость относительного коэффициента теплопроводности $\lambda_{r}=\lambda / \lambda_{f}$ той же наножидкости, что и на рис. 1 , от объемной концентрации наночастиц $(\rho=0.707$, а $T=300 \mathrm{~K})$ представлена на рис. 2. Здесь треугольниками отмечены данные моделирования, а штриховая линия 2 соответствует формуле (1). Теплопроводность рассматриваемой наножидкости существенно превосходит теплопроводность и базовой жидкости, и крупнодисперсных жидкостей (1). Так, теплопроводность двухпроцентной наножидкости почти вдвое превосходит значение, определяемое формулой (1).

Зависимость коэффициента теплопроводности наножидкости при небольшой концентрации (до 10-15\%) наночастиц обычно хорошо описывается квадратичной зависимостью от их объемной концентрации $\varphi$ вида

$$
\lambda_{r}=\lambda / \lambda_{f}=1+a_{1} \varphi-a_{2} \varphi^{2} .
$$

Формуле (10) на рис. 2 соответствует пунктирная линия 1 , причем в данном случае $a_{1}=63.1, a_{2}=-607.9$. Наличие в (10) второго члена с отрицательным знаком указывает на то, что с ростом концентрации теплопроводность наножидкости выходит на некоторое предельное значение. Как уже указывалось, это наблюдается и экспериментально. С другой стороны, при малых концентрациях наночастиц (когда вторым членом в формуле (10) можно пренебречь) теплопроводность растет линейно с концентрацией. Здесь относительное превышение теплопроводности $\Delta=\left(\lambda_{r}-1\right)$ более чем в 20 раз превосходит значение, предсказываемое теорией Максвелла.

Как следует из (5), коэффициент теплопроводности наножидкости является суперпозицией двух членов. Если первое слагаемое $\lambda_{0}$ присутствует как в чистой жидкости (однокомпонентной среде), так и в наножидкости, то второе слагаемое $\lambda_{d}-$ только в наножидкости. Зависимость этих членов от объемной концентрации частиц представлена на рис. 3. Здесь треугольники соответствуют данным расчета, а пунктирные линии - их 
аппроксимация вида (10). Вклад диффузионного члена невелик и, начиная с некоторой концентрации, он снижается. Последнее совершенно естественно, с ростом концентрации наночастиц их диффузия уменьшается.

Чтобы ответить на вопрос, какова зависимость коэффициента теплопроводности от диаметра наночастиц, рассматривались две наножидкости на основе аргона с наночастицами из цинка и алюминия. В обоих случаях объемная концентрация наночастиц равнялась $4.2 \%$, плотность несущего флюида $\rho=0.707$, а температура $T=300 \mathrm{~K}$. Полученные данные приведены на рис. 4 . Здесь квадратики и линия 1 соответствуют данным для наножидкости с частицами из цинка, треугольники и линия 2 - наножидкости с частицами из алюминия, линия 3 - значение, рассчитанное по формуле (1) для суспензий при той же объемной концентрации частиц, штрихпунктирная линия 4 - данные для чистого аргона. Приведенные данные для этих двух наножидкостей радикально разнятся. Наножидкость с частицами цинка во всех случаях имеет теплопроводность существенно выше теплопроводности и несущей жидкости, и значения, определяемого формулой (1). Напротив, наножидкость с частицами алюминия размером $1 \mathrm{~nm}$ оказывается ниже теплопроводности несущей жидкости. Но наножидкость
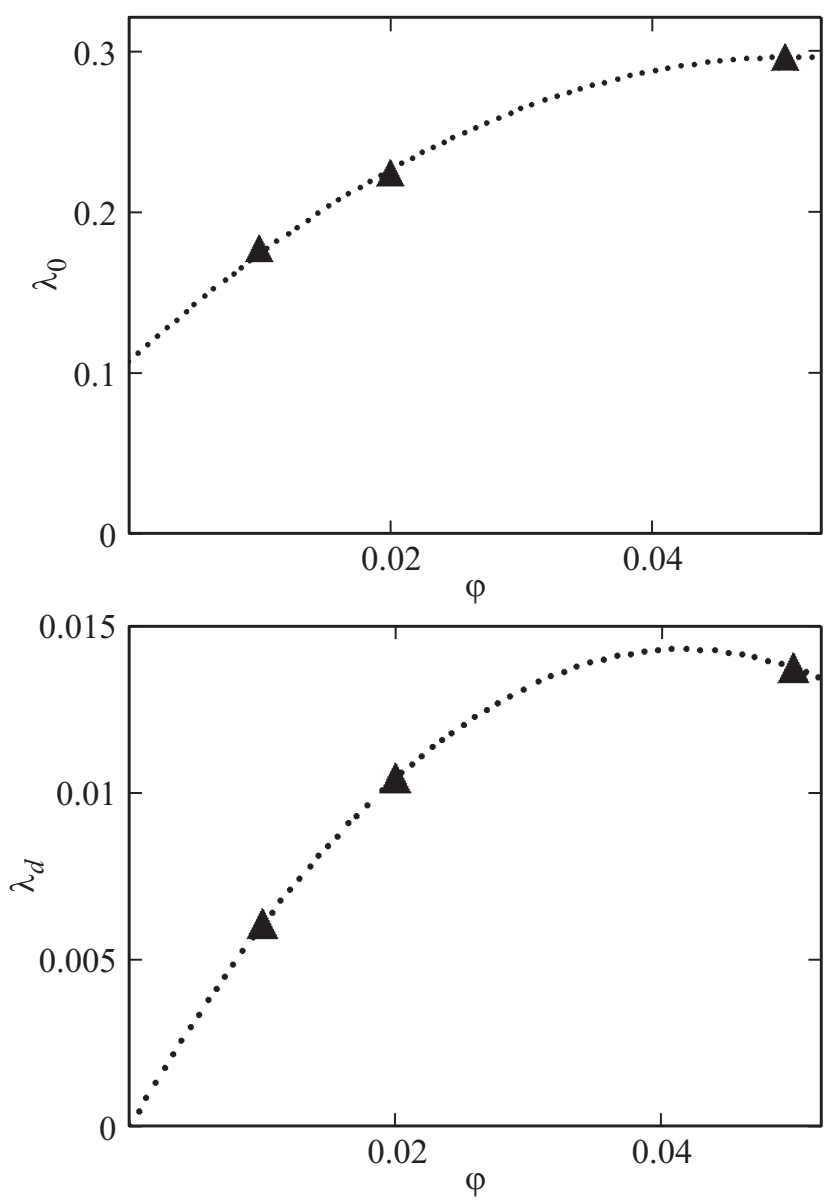

Рис. 3. Зависимость коэффициентов $\lambda_{0}$ и $\lambda_{d}(\mathrm{~W} / \mathrm{mK})$ от объемной концентрации наночастиц $(d=2 \mathrm{~nm})$.

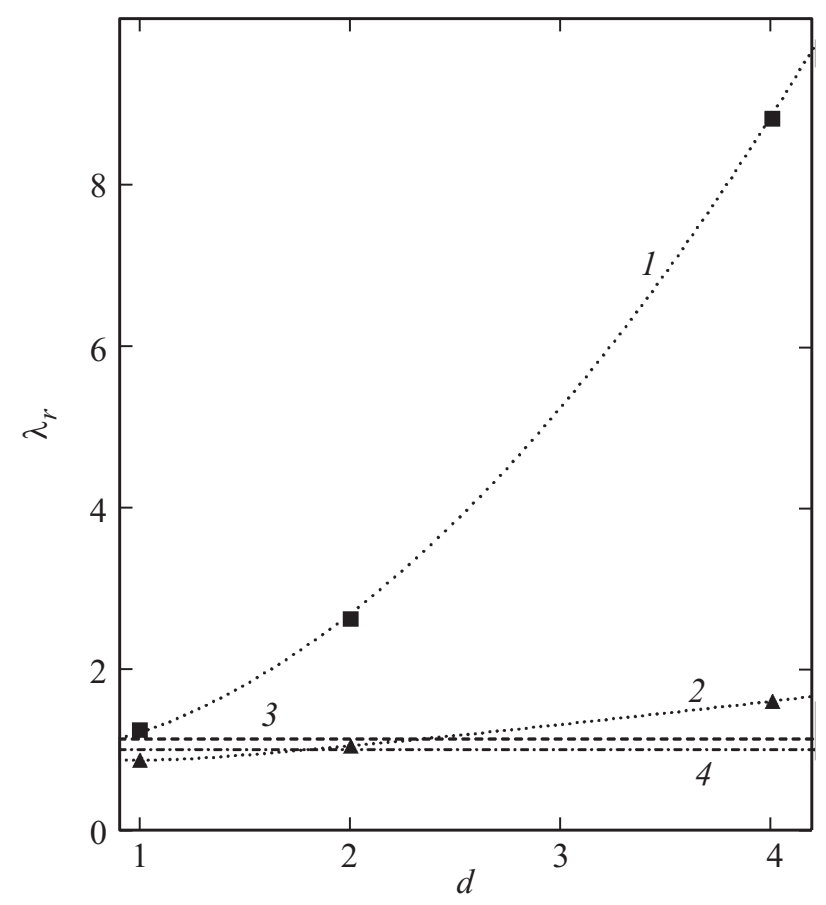

Рис. 4. Зависимость относительного коэффициента теплопроводности наножидкостей $\mathrm{Ar}-\mathrm{Zn}$ и $\mathrm{Ar}-\mathrm{Al}$ от диаметра наночастиц $(\mathrm{nm})$.

с частицами $4 \mathrm{~nm}$ уже превосходит значение, определяемое формулой (1).

В классических теориях теплопроводности наножидкостей материал дисперсных частиц учитывается через их теплопроводность (см. формулу (1)). Однако в наножидкостях с металлическими наночастицами это не так. Такие частицы имеют теплопроводность на порядки превосходящую теплопроводность несущей жидкости: $\lambda_{f} \ll \lambda_{p}$, и в этом случае формула (1) перестает зависеть от материала частиц и принимает совсем простой вид: $\lambda_{r}=(1+1 \phi) /(1-\phi)$. Таким образом, классические теории фактически не дают зависимости коэффициента теплопроводности дисперсных жидкостей от материала частиц. Тем не менее представленные данные показывают, что такая зависимость есть. Так, например, коэффициент теплопроводности для наножидкости $\mathrm{Ar}-\mathrm{Zn} \mathrm{c}$ наночастицами цинка диаметра $2 \mathrm{~nm}$ и объемной концентрацией 4.2\% примерно в 2.4 больше соответствующего значения для наножидкости Ar-Al. Но плотность цинка также больше плотности алюминия. Таким образом, можно утверждать, что теплопроводность наножидкости зависит от материала наночастиц, и она растет с увеличением плотности материала наночастиц.

\section{Анализ различных вкладов в коэффициент теплопроводности}

Анализ формул (6)-(9) показывает, что коэффициент теплопроводности определяется рядом разнородных 
вкладов, переносом тепла в результате движения атомов флюида и наночастиц, их взаимодействия и т.п. Чтобы выяснить причину существенного превышения теплопроводности наножидкости над соответствующим значением для базовой жидкости, необходимо проанализировать все возможные составляющие. Вклад в коэффициент теплопроводности $\lambda_{0}(5)$ является суммой шести слагаемых:

$$
\begin{gathered}
\lambda_{0}=\lambda_{0}^{\mathrm{K}}+\lambda_{0}^{\mathrm{P}}+\lambda_{0}^{\mathrm{C}}+\lambda_{0}^{\mathrm{KP}}+\lambda_{0}^{\mathrm{KC}}+\lambda_{0}^{\mathrm{PC}}, \\
\lambda_{0}^{\mathrm{K}}=\frac{V}{3 k_{B} T^{2}} \int_{0}^{\tau} \chi_{0}^{\mathrm{K}}(t) d t=\frac{V}{3 k_{B} T^{2}} \int_{0}^{\tau} \mathbf{j}_{Q}^{\mathrm{K}}(0) \mathbf{j}_{Q}^{\mathrm{K}}(t) d t, \\
\lambda_{0}^{\mathrm{P}}=\frac{V}{3 k_{B} T^{2}} \int_{0}^{\tau} \chi_{0}^{\mathrm{P}}(t) d t=\frac{V}{3 k_{B} T^{2}} \int_{0}^{\tau} \mathbf{j}_{Q}^{\mathrm{P}}(0) \mathbf{j}_{Q}^{\mathrm{P}}(t) d t, \\
\lambda_{0}^{\mathrm{C}}=\frac{V}{3 k_{B} T^{2}} \int_{0}^{\tau} \chi_{0}^{\mathrm{C}}(t) d t=\frac{V}{3 k_{B} T^{2}} \int_{0}^{\tau} \mathbf{j}_{Q}^{\mathrm{C}}(0) \mathbf{j}_{Q}^{\mathrm{C}}(t) d t, \\
\lambda_{0}^{\mathrm{KP}}=\frac{2 V}{3 k_{B} T^{2}} \int_{0}^{\tau} \chi_{0}^{\mathrm{KP}}(t) d t=\frac{2 V}{3 k_{B} T^{2}} \int_{0}^{\tau} \mathbf{j}_{Q}^{\mathrm{K}}(0) \mathbf{j}_{Q}^{\mathrm{P}}(t) d t, \\
\lambda_{0}^{\mathrm{KC}}=\frac{2 V}{3 k_{B} T^{2}} \int_{0}^{\tau} \chi_{0}^{\mathrm{KC}}(t) d t=\frac{2 V}{3 k_{B} T^{2}} \int_{0}^{\tau} \mathbf{j}_{Q}^{\mathrm{K}}(0) \mathbf{j}_{Q}^{\mathrm{C}}(t) d t, \\
\frac{2 V}{3 k_{B} T^{2}} \int_{0}^{\tau} \chi_{0}^{\mathrm{PC}}(t) d t=\frac{2 V}{3 k_{B} T^{2}} \int_{0}^{\tau} \mathbf{j}_{Q}^{\mathrm{P}}(0) \mathbf{j}_{Q}^{\mathrm{C}}(t) d t .
\end{gathered}
$$

Аналогично можно представить и диффузионный вклад $\lambda_{d}(5)$

$$
\begin{aligned}
& \lambda_{d}= \lambda_{d}^{\mathrm{K}}+\lambda_{d}^{\mathrm{P}}+\lambda_{d}^{\mathrm{C}}+\lambda_{d}^{\mathrm{KP}}+\lambda_{d}^{\mathrm{KC}}+\lambda_{d}^{\mathrm{PC}} \\
&= \frac{1}{T^{2} L_{22}}\left[\left(L_{2}^{\mathrm{K}}\right)^{2}+\left(L_{2}^{\mathrm{P}}\right)^{2}+\left(L_{2}^{\mathrm{C}}\right)^{2}+2 L_{2}^{\mathrm{K}} L_{2}^{\mathrm{P}}\right. \\
&\left.+2 L_{2}^{\mathrm{K}} L_{2}^{\mathrm{C}}+2 L_{2}^{\mathrm{P}} L_{2}^{\mathrm{C}}\right] \\
& L_{2}^{\mathrm{K}}=\frac{V}{3} \int_{0}^{T}\left\langle\mathbf{j}_{Q}^{\mathrm{K}}(0) \mathbf{j}_{d 2}(t)\right\rangle d t, \quad L_{2}^{\mathrm{P}}=\frac{V}{3} \int_{0}^{T}\left\langle\mathbf{j}_{Q}^{\mathrm{P}}(0) \mathbf{j}_{d 2}(t)\right\rangle d t \\
& L_{2}^{\mathrm{C}}=\frac{V}{3} \int_{0}^{T}\left\langle\mathbf{j}_{Q}^{\mathrm{C}}(0) \mathbf{j}_{d 2}(t)\right\rangle d t
\end{aligned}
$$

В результате можно вычислить все шесть вкладов в коэффициент теплопроводности наножидкости: кинетический (индекс $\mathrm{K}$ ), потенциальный $(\mathrm{P})$, столкновительный $(\mathrm{C})$, потенциально-кинетический $(\mathrm{KP})$, столкновительно-кинетический $(\mathrm{KC})$, потенциальностолкновительный (РС)

$$
\begin{gathered}
\lambda^{\mathrm{K}}=\lambda_{0}^{\mathrm{K}}-\lambda_{d}^{\mathrm{K}}=\frac{1}{T^{2}}\left[L_{0}^{\mathrm{K}}-\frac{\left(L_{2}^{\mathrm{K}}\right)^{2}}{L_{22}}\right], \\
\lambda^{\mathrm{P}}=\lambda_{0}^{\mathrm{P}}-\lambda_{d}^{\mathrm{P}}=\frac{1}{T^{2}}\left[L_{0}^{\mathrm{P}}-\frac{\left(L_{2}^{\mathrm{P}}\right)^{2}}{L_{22}}\right], \\
\lambda^{\mathrm{C}}=\lambda_{0}^{\mathrm{C}}-\lambda_{d}^{\mathrm{C}}=\frac{1}{T^{2}}\left[L_{0}^{\mathrm{C}}-\frac{\left(L_{2}^{\mathrm{C}}\right)^{2}}{L_{22}}\right], \\
\lambda^{\mathrm{KP}}=\lambda_{0}^{\mathrm{KP}}-\lambda_{d}^{\mathrm{KP}}=\frac{1}{T^{2}}\left[L_{0}^{\mathrm{KP}}-2 \frac{L_{2}^{\mathrm{K}} L_{2}^{\mathrm{P}}}{L_{22}}\right], \\
\lambda^{\mathrm{KC}}=\lambda_{0}^{\mathrm{KC}}-\lambda_{d}^{\mathrm{KC}}=\frac{1}{T^{2}}\left[L_{0}^{\mathrm{KC}}-2 \frac{L_{2}^{\mathrm{K}} L_{2}^{\mathrm{C}}}{L_{22}}\right], \\
\lambda^{\mathrm{PC}}=\lambda_{0}^{\mathrm{PC}}-\lambda_{d}^{\mathrm{PC}}=\frac{1}{T^{2}}\left[L_{0}^{\mathrm{PC}}-2 \frac{L_{2}^{\mathrm{C}} L_{2}^{\mathrm{P}}}{L_{22}}\right] .
\end{gathered}
$$

Результаты расчетов для аргона и наножидкостей на основе аргона с частицами цинка диаметрами 2 и $4 \mathrm{~nm}$ при их объемной концентрации $\varphi=4.2 \%$ и массовой концентрации $C_{2}=0.208$ приведены в таблице. Давление наножидкостей равнялось давлению чистого Ar при плотности $\rho=0.707$ и температуре $T=300 \mathrm{~K}$. Основной вклад в коэффициент теплопроводности чистого аргона дают коэффициенты $L^{\mathrm{C}}(62 \%)$, определяющие поток энергии, связанный со столкновениями атомов аргона друг с другом, и $L^{\mathrm{KC}}(22 \%)$, обусловленный корреляцией потока кинетической энергии и потока энергии в результате столкновений. Это естественно, поскольку плотность аргона велика. Кинетический переноса тепла, обусловленный движением молекул $\left(L^{\mathrm{K}}\right)$, здесь невелик и составляет всего около $8 \%$.

Чем отличается структура теплопроводности наножидкости по сравнению с чистым аргоном? Как видно из таблицы, наряду с положительными вкладами в рассматриваемых бинарных системах появляются и отрицательные. Физически это означает, что наряду с обычным потоком тепла появляется и поток, согласованный с градиентом температуры (отрицательная теплопроводность). Как и для чистой жидкости, столкновительный вклад $\lambda^{\mathrm{C}}$ является важным, и он увеличивается для наножидкостей (в 1.5 раза для наножидкости с диаметром наночастиц $2 \mathrm{~nm}$ и в 3.6 раза для наножидкости с диаметром наночастиц $4 \mathrm{~nm})$. Однако наибольший вклад в теплопроводность обусловлен членом $\lambda^{\mathrm{KC}}$. Он составляет от $50 \%$ для наножидкости с частицами $2 \mathrm{~nm}$ до почти $80 \%$ для наножидкости с частицами $4 \mathrm{~nm}$ от полного значения коэффициента теплопроводности. Значительным оказывается также и кинетический вклад $\lambda^{\mathrm{K}}$, он составляет $34 \%$ для наножидкости с диаметром наночастиц $2 \mathrm{~nm}$ и $45 \%$ для наножидкости с диаметром наночастиц $4 \mathrm{~nm}$ от полного значения $\lambda$. Таким образом, рост теплопроводности наножидкости по сравнению с чистой жидкостью обусловлен в основном значительным увеличением кинетического $\lambda^{\mathrm{K}}$ и кинетическостолкновительного $\lambda^{\mathrm{KC}}$ вкладов. 


\section{Эволюция корреляционных функций}

Для оценки различных механизмов повышения теплопроводности в наножидкости полезно знать не только величину различных вкладов, но и динамику соответствующих корреляционных функций. Эволюция корреляционных функций флуктуаций потока тепла, дающих наибольший вклад в коэффициент теплопроводности, $\chi_{0}^{\mathrm{K}}(t), \chi_{0}^{\mathrm{C}}(t), \chi_{0}^{\mathrm{KC}}(t) \quad$ (см. формулы (11)) представлена на рис. 5. Здесь слева приведены данные для аргона, а справа - для наножидкости на основе аргона с частицами цинка диаметром $2 \mathrm{~nm}(\varphi=4.2 \%)$. Корреляционные функции $\chi(t)$ нормированы на величину $\chi^{\mathrm{K}}(0)$ для чистого аргона, время - на $\tau_{0}$. Корреляционной функции $\chi_{0}^{\mathrm{K}}(t)$ соответствует линия $1, \chi_{0}^{\mathrm{C}}(t)-$ линия 2 , $\chi_{0}^{\mathrm{KC}}(t)$ - линия 3. Для чистого аргона все рассмотренные корреляционные функции затухают экспоненциально быстро с характерным временем порядка времени взаимодействия молекул. Эволюция корреляционных функций для наножидкостей сложнее. Как показано в [26], автокорреляционная функция скорости (АКФС) наночастицы является суперпозицией двух экспонент. Время релаксации первой порядка времени взаимодействием наночастицы с отдельными молекулами несущего флюида, а второй - порядка времени взаимодействия наночастицы с микрофлуктуациями гидродинамических полей несущей среды. Это последнее время обычно в несколько раз больше первого. Аналогично ведут себя и корреляционные функции, определяющие основные вклады в теплопроводность наножидкости. Их эволюция также двухстадийная. На первой стадии время релакса-

Вклады различных слагаемых в коэффициент теплопроводности наножидкости, $\mathrm{W} /(\mathrm{mK})$

\begin{tabular}{c|c|c|c}
\hline$\lambda$ & $\mathrm{Ar}$ & $\mathrm{Ar}-\mathrm{Zn},(d=2 \mathrm{~nm})$ & $\mathrm{Ar}-\mathrm{Zn},(d=4 \mathrm{~nm})$ \\
\hline$\lambda_{0}$ & & 0.282163011 & 0.807266812 \\
$\lambda_{d}$ & & 0.0131916228 & 0.0117404684 \\
$\lambda$ & 0.10680659 & 0.268971388 & 0.795526344 \\
$\lambda_{0}^{\mathrm{K}}$ & & 0.13939356 & 0.555674513 \\
$\lambda_{d}^{\mathrm{K}}$ & & 0.0478870749 & 0.199758098 \\
$\lambda^{\mathrm{K}}$ & 0.00853276 & 0.0915064848 & 0.355916415 \\
$\lambda_{0}^{\mathrm{C}}$ & & 0.0965082435 & 0.235437078 \\
$\lambda_{d}^{\mathrm{C}}$ & & 0.0023135801 & 0.000153929297 \\
$\lambda^{\mathrm{C}}$ & 0.06503381 & 0.0941946634 & 0.235283149 \\
$\lambda_{0}^{\mathrm{P}}$ & & 0.0163644013 & 0.10063356 \\
$\lambda_{d}^{\mathrm{P}}$ & & 0.0231271266 & 0.123198502 \\
$\lambda^{\mathrm{P}}$ & 0.00050646 & -0.00676272531 & -0.0225649413 \\
$\lambda_{0}^{\mathrm{KC}}$ & & 0.156042641 & 0.63243097 \\
$\lambda_{d}^{\mathrm{K}}$ & & 0.0210514212 & 0.0110902883 \\
$\lambda^{\mathrm{K}}$ & 0.02352531 & 0.13499122 & 0.621340682 \\
$\lambda_{0}^{\mathrm{KP}}$ & & -0.083342968 & -0.464380266 \\
$\lambda_{d}^{\mathrm{KP}}$ & & -0.0665579581 & -0.313750846 \\
$\lambda^{\mathrm{KP}}$ & 0.00262643 & -0.02207763387 & -0.15062942 \\
$\lambda_{0}^{\mathrm{KC}}$ & & -0.0388115389 & -0.252529043 \\
$\lambda_{0}^{\mathrm{PC}}$ & & -0.014629622 & -0.00870950256 \\
$\lambda^{\mathrm{PC}}$ & 0.00658181 & -0.0241819169 & -0.243819541 \\
& & &
\end{tabular}

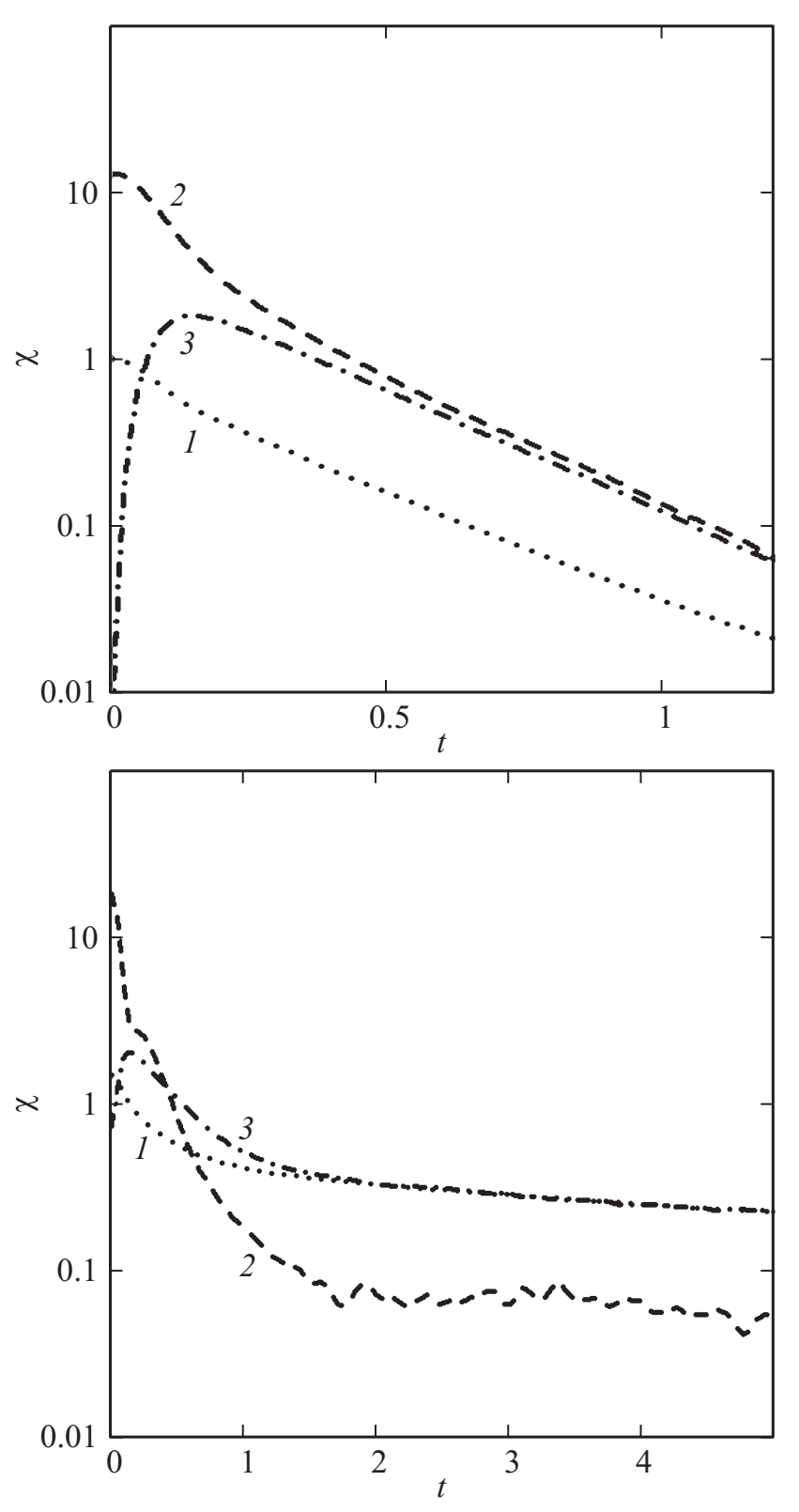

Рис. 5. Корреляционные функции флуктуаций потока тепла в чистом аргоне и в наножидкости Ar-Zn.

ции равно времени релаксации корреляционных функций коэффициента теплопроводности несущего флюида, а начиная со времен примерно $2 \tau_{0}$, описывается некоторым временем релаксации $\tau_{f}$.

Рассмотрим более подробно механизмы релаксации АКФС наночастицы. Кинетический механизм будет тем эффективнее, чем меньше размеры и масса частиц. Уже для частиц размером 1-2nm его вклад в общую релаксацию корреляционных функций не велик. Начиная с этого размера, релаксация корреляционных функций наночастицы будет осуществляться преимущественно за счет ее взаимодействия с микрофлуктуациями гидродинамических полей несущей среды. Как показано в работах $[27,28]$, характерный масштаб этих флуктуа- 
ций $l_{f}$ порядка размера самой наночастицы (по крайней мере, для не слишком крупных частиц). Поэтому время $\tau_{f}$ должно быть порядка времени „взаимодействия“ дисперсной частицы с флуктуацией, которое в свою очередь определяется соотношением $\tau_{f} \sim l_{f} / c_{p} \sim R / c_{p}$, здесь $c_{p}$ - средняя тепловая скорость наночастицы. Это время легко связать со временем взаимодействия молекул

$$
\tau_{f} \sim \frac{R}{c_{p}} \sim \frac{R}{c} \sqrt{\frac{M}{m}} \sim \frac{R}{\sigma} \sqrt{\frac{M}{m}} \tau_{0},
$$

где $m, M-$ соответственно масса молекулы несущей жидкости и наночастицы.

Для рассматриваемых наночастиц время (14) по крайней мере на порядок больше, чем характерное время релаксации молекул несущей жидкости $\tau_{0}$. Это, в частности, означает, что характерное затухание всех корреляционных функций, определяющих коэффициент теплопроводности наножидкости, на порядок больше, чем для базового флюида. Расчеты именно это и фиксируют (рис. 5). Следствием медленной релаксации этих корреляционных функций и является значительный рост коэффициента теплопроводности наножидкостей по сравнению с соответствующими значениями для базовой жидкости. Из оценки (14) также следует, что время релаксации $\tau_{f}$, а значит и величина коэффициента теплопроводности, будет расти с увеличением размера наночастиц и их плотности (массы).

\section{Заключение}

Выполненное молекулярно-динамическое моделирование прежде всего позволяет утверждать, что коэффициент теплопроводности наножидкостей не описывается формулой Максвелла (1) (или ее обобщениями). Коэффициент теплопроводности наножидкостей с металлическими частицами не зависит от их теплопроводности и в общем случае может существенно превосходить значение, определяемое формулой (1). Он увеличивается с ростом концентрации частиц, но затем достигает некоторого предельного значения, после чего меняется слабо. В отличие от всех классических теорий коэффициент теплопроводности наножидкости зависит также от размера наночастиц, причем он растет с его увеличением. Вместе с тем могут существовать ситуации, когда при достаточно малом размере частиц теплопроводность наножидкостей оказывается не выше (или даже ниже) значений, предсказываемых теорией Максвелла. Однако при дальнейшем повышении размера наночастиц теплопроводность наножидкости последовательно увеличивается и затем становится выше значений, предсказываемых формулой (1). Эффект превышения коэффициента теплопроводности помимо размера наночастиц определяется и плотностью их материала. По-видимому, это превышение (в частности и снижение) зависит от соотношения плотности материала частицы и несущей жидкости, на что указывали и данные молекулярнодинамического моделирования для системы твердых сфер [29]. В этой связи полезно отметить, что с ростом плотности материала частиц резко возрастает их массовая концентрация в наножидкости. Так, например, в исследованных наножидкостях на основе аргона с алюминиевыми наночастицами при их фиксированной объемной концентрации, равной 4.2\%, массовая доля частиц алюминия составляла 0.09 , а частиц цинка 0.208. Таким образом, превышение теплопроводности наножидкости над соответствующим значением для базовой жидкости тем выше, чем больше массовая доля частиц.

В настоящее время нет общепринятой модели теплопроводности наножидкостей, хотя попыток их построения было много (см. обзор [12] и цитированную там литературу). Более того, фактически нет понимания причин необычного повышения теплопроводности по сравнению с обычной дисперсной жидкостью. Различные возможные причины обсуждались многими авторами. Возможно, наиболее систематически это было сделано в работах Кеблинского с коллегами $[30,31]$ и в обзоре Кляйнстройера и Фенга [32]. В них анализировался вклад четырех возможных механизмов. Рассматривалось влияние (i) баллистического фононного переноса тепла, (ii) образования слоя жидкости вокруг наночастиц, имеющего повышенную теплопроводность, (iii) броуновского движения наночастиц, (iv) кластеризации наночастиц. Баллистический фононный механизм создания дополнительного превышения теплопроводности справедливо отвергается практически всеми авторами. В нашем моделировании этот механизм не учитывается, так как мы считаем наночастицы твердыми, внутри которых не происходит передачи энергии.

Теперь относительно кластеризации наночастиц. В принципе наличие кластеризации наночастиц могло бы привести к увеличению теплопроводности. Но надо иметь в виду следующее обстоятельство. Если процесс кластеризации имеет место, то в жидкости появляются частицы с макроскопическими размерами. Но тогда, с одной стороны, теплопроводность должна перестать зависеть от размера наночастиц (как в классических теориях), а с другой, такие частицы должны быстро седиментировать. Ни того, ни другого в хорошо поставленных экспериментах не наблюдается. Хорошо поставленных означает, что, по крайней мере, за время серии измерений, средний размер наночастиц остается постоянным и практически не меняется распределение частиц по размерам. Такие наножидкости, безусловно, и используются в реальных экспериментах. На это же указывает и молекулярно динамические расчеты для системы твердых сфер [29], где кластеризация не возможна в принципе, тем не менее заметный рост теплопроводности по сравнению с теорией Максвелла был зафиксирован. На это же указывают и наши расчеты при использовании потенциала мягких сфер для описания взаимодействия наночастиц. 
Броуновское движение наночастиц неоднократно обсуждалось в качестве одного из механизмов повышения теплопроводности наножидкостей по сравнению с чистыми жидкостями. Прямое влияние броуновского движения наночастиц на теплопроводности наножидкости, как правильно и указывается в [30,31], не велико. Однако наножидкость - это бинарная система частиц, в которой имеет место активная взаимная диффузия компонентов. Наличие формирующихся при этом потоков, по-видимому, и определяет основной механизм теплопроводности наножидкостей. Диффузионное движение наночастиц и молекул приводит к тому, что вклад в теплопроводность, обусловленный потоками переносимой при этом кинетической энергии, может возрастать на порядок и более.

Как уже выше указывалось, вокруг наночастиц действительно формируется слой молекул несущей жидкости, параметры которой скоррелированы с частицей. Наличие таких слоев упорядоченной жидкости будет существенно изменять поток тепла, обусловленный переносом кинетической и потенциальной энергий молекул, движущихся вместе с наночастицей. В таблице этому соответствуют члены $\lambda^{\mathrm{K}}, \lambda^{\mathrm{P}}$ и $\lambda^{\mathrm{KP}}$. Такой перенос в наножидкости действительно может возрастать больше чем на порядок и вносить существенный вклад в теплопроводность наножидкости (в наших расчетах вклад $\lambda^{\mathrm{K}}$ составляет до $45 \%$ ).

Работа выполнена при частичной поддержке РФФИ (грант № 17-01-00040).

\section{Список литературы}

[1] Masuda H. et al. // Netsu Bussei (Japan). 1993. Vol. 4. P. $227-$ 239.

[2] Wang X., Xu X., Choi S.U.S. // J. Thermophys. Heat Trans. 1999. Vol. 13. N 4. P. 474-480.

[3] Wong K.V., De Leon O. // Adv. Mechanic. Eng. 2010. Article ID 519659.

[4] Rudyak V.Ya., Belkin A.A., Tomilina E.A., Egorov V.V. // Defect and Diffusion Forum. 2008. Vol. 273-276. P. 566-571.

[5] Timofeeva E.V., Smith D.S., Yu W., France D.M., Singh D., Routbo J.L. // Nanotechnology. 2010. Vol. 21. P. 215703.

[6] Mahbubul I.M., Saidur R., Amalina M.A. // Int. J. Heat and Mass Transfer. 2012. Vol. 55. P. 874-885.

[7] Rudyak V.Ya. // Advan. Nanoparticles. 2013. Vol. 2. P. 266 279.

[8] Rudyak V.Ya., Krasnolutskii S.L. // Phys. Lett. A. 2014. Vol. 378. P. 1845-1849.

[9] Рудяк В.Я. // Вестн. НГУ. Серия: Физика. 2015. Т. 10. Вып. 1. C. 5-22.

[10] Zhu H.T, Zhang C.Y., Tang Y.M., Wang J.X. // J. Phys. Chem. C. 2007. Vol. 111. P. 1646-1650.

[11] Rudyak V., Belkin A., Tomilina E. // In book: Proc. $3^{\text {rd }}$ European Conf. on Microfluidics. Heidelberg: SHF. 2012. $\mu$ Flu-152.

[12] Kumar P.M., Kumar J., Tamilarasan R., Sendhilnathan1 S., Suresh S. // Engineering J. 2015. Vol. 19. N 1. P. 67-83.
[13] Eapen J., Li J., Yip S. // Phys. Rev. Lett. 2007. Vol. 98. P. 028302.

[14] Rudyak V.Ya., Krasnolutskii S.L., Ivanov D.A. // Microfluid. Nanofluid. 2011. Vol. 11. N 4. P. 501-506.

[15] Rudyak V.Ya., Krasnolutskii S.L. // Phys. Lett. A. 2014. Vol. 378. P. 1845-1849.

[16] Рудяк В.Я., Краснолучякий С.Л. // ЖТФ. 2015. Т. 85. Вып. 6. C. 9-16.

[17] Рудяк В.Я., Краснолуиякий С.Л. // ЖТФ. 2002. Т. 72. Вып. 7. C. $13-20$.

[18] Рудяк В.Я., Краснолуцкий С.Л., Иванов Д.А. // ДАН. 2012. T. 442. № 1. C. 54-56.

[19] Гиршфельдер Джс., Кертисс Ч., Бёрд Р. Молекулярная теория газов и жидкостей. М.: ИИЛ, 1961. 929 с.

[20] Арефбев К.М. Явления переноса в газе и плазме. Л.: Энергоатомиздат, 1983. $127 \mathrm{c.}$

[21] Heinz H., Vaia R.A., Farmer B.L., Naik R.R. // J. Phys. Chem. C. 2008. Vol. 112. N 44. P. 17281-17290.

[22] Рудяк В.Я., Иванов Д.А. // ДАН ВШ. 2003. № 1. С. 30-38.

[23] Норман Г.Э., Стегайлов В.В. // Мат. моделирование. 2012. T. 24. № 6. C. 3-44.

[24] Зубарев Д.Н. Неравновесная статистическая термодинамика. М.: Наука, 1971. 416 с.

[25] Рудяк В.Я., Белкин А.А., Иванов Д.А., Егоров В.В. // ТВТ. 2008. T. 46. № 1. C. 35-44.

[26] Рудяк В.Я., Харламов Г.В., Белкин А.А. // Письма в ЖТФ. 2000. Т. 26. Вып. 13. С. 29-36.

[27] Рудяк В.Я., Белкин А.А. // Письма в ЖТФ. 2003. Т. 29. Вып. 13. С. 71-79.

[28] Рудяк В.Я., Белкин А.А. // Теплофизика и аэромеханика. 2004. T. 11. № 2. C. 249-259.

[29] Рудяк В.Я., Белкин А.А., Томилина Е.А. // Письма в ЖТФ. 2010. Т. 36. Вып. 14. С. 49-54.

[30] Keblinski P., Philpot S.R., Choi S.U.S., Eastman J.A. // Int. J. Heat Mass Transfer. 2002. Vol. 45. P. 855-863.

[31] Keblinski P., Prasher R., Eapen J.T // J. Nanoparticle Research. 2008. Vol. 10. N 7. P. 1089-1097.

[32] Kleinstreuer C., Feng Y. // Nanoscale Research Lett. 2011. Vol. 6. P. 229-240. 